Research, Society and Development, v. 9, n. 10, e9989109477, 2020

(CC BY 4.0) | ISSN 2525-3409 | DOI: http://dx.doi.org/10.33448/rsd-v9i10.9477

\title{
O papel do enfermeiro na assistência, educação e promoção da saúde no pré-natal
}

The role of nurses in prenatal care, education and health promotion

El papel de las enfermeras en la atención prenatal, la educación y la promoción de la salud

Recebido: 20/10/2020 | Revisado: 24/10/2020 | Aceito: 27/10/2020 | Publicado: 30/10/2020

Ana Alice Bueno Da Silva

ORCID: https://orcid.org/0000-0001-7413-3824

Faculdade Cristo Rei, Brasil

E-mail: anaalicecp98@hotmail.com

Claudiane Andrade

ORCID: https://orcid.org/0000-0002-9760-0090

Faculdade Cristo Rei, Brasil

E-mail: claudiane@ faccrei.edu.br

\section{Resumo}

O acompanhamento pré-natal é fundamental para a manutenção da saúde da gestante e do feto, sendo assessorado por um aparato multiprofissional. Os enfermeiros destacam-se na promoção e educação em saúde ao longo das consultas do pré-natal, amparando as gestantes acerca dos cuidados e riscos gestacionais. Este trabalho teve como objetivo investigar por meio de uma revisão integrativa da literatura a atuação dos enfermeiros na educação e na promoção de saúde no pré-natal. A pesquisa foi realizada de forma descritiva, por meio de uma revisão integrativa da literatura nas bases de dados da Biblioteca Virtual em Saúde (BVS) e na SciELO (Eletronic Library Online), entre os anos de 2010-2020. Os resultados demostraram aprimoramento na atuação do enfermeiro no acompanhamento pré-natal, com estabelecimento de vínculos junto as gestantes e elaboração de ações que promovem a educação da gestante, sobretudo, frente a aspectos como amamentação, alimentação e atividade física. Contudo, concluímos que tais ações ainda são limitadas e carecem de aperfeiçoamento cientifico, afim de os enfermeiros atuem plenamente em prol da efetivação e qualificação do pré-natal.

Palavras-chave: Pré-natal; Enfermagem; Consulta da enfermagem. 


\begin{abstract}
Prenatal care is essential for maintaining the health of the pregnant woman and the fetus, being assisted by a multiprofessional apparatus. Nurses stand out in health promotion and education throughout prenatal consultations, supporting pregnant women about gestational care and risks. This study aimed to investigate through an integrative literature review the role of nurses in education and health promotion in prenatal care. The research was carried out in a descriptive manner, through an integrative literature review in the databases of the virtual health library (vhl) and scielo (electronic library online), between the years 2010-2020. The results showed an improvement in the performance of nurses in prenatal care, with the establishment of bonds with pregnant women and the development of actions that promote the education of pregnant women, especially in the face of aspects such as breastfeeding, food and physical activity. However, we conclude that such actions are still limited and need scientific improvement, in order for nurses to act fully in favor of the effectiveness and qualification of prenatal care.
\end{abstract}

Keywords: Prenatal; Nursing; Nursing consultation.

\title{
Resumen
}

La atención prenatal es fundamental para mantener la salud de la gestante y del feto, siendo asistida por un aparato multiprofesional. Las enfermeras se destacan en la promoción y educación de la salud a lo largo de las consultas prenatales, apoyando a las embarazadas sobre los cuidados y riesgos gestacionales. Este estudio tuvo como objetivo investigar a través de una revisión integradora de la literatura el papel de las enfermeras en la educación y promoción de la salud en la atención prenatal. La investigación se realizó de manera descriptiva, a través de una revisión integrativa de la literatura en las bases de datos de la Biblioteca Virtual en Salud (BVS) y SciELO (Biblioteca Electrónica en Línea), entre los años 2010-2020. Los resultados mostraron una mejora en el desempeño de las enfermeras en el cuidado prenatal, con el establecimiento de vínculos con la gestante y el desarrollo de acciones que promuevan la educación de la gestante, especialmente ante aspectos como la lactancia materna, la alimentación y la actividad física. Sin embargo, llegamos a la conclusión de que estas acciones aún son limitadas y necesitan una mejora científica, para que las enfermeras actúen plenamente a favor de la efectividad y calificación de la atención prenatal.

Palabras clave: Prenatal; Enfermería; Consulta de enfermería. 


\section{Introdução}

O pré-natal representa uma etapa crucial de cuidados com a saúde durante a gravidez. O acompanhamento continuo dos profissionais da saúde, por meio de consultas e exames promove o diagnóstico rápido dos fatores de risco associados a saúde da gestante e do bebê, além de amparar as futuras mamães com instruções importantes acerca dos cuidados e riscos relacionados a este período.

O Ministério da Saúde do Brasil recomenda para o Sistema Único de Saúde (SUS) e para a rede hospitalar privada que as gestantes deem início ao pré-natal ainda no primeiro trimestre gestacional, com a realização mínima de 6 consultas, ademais, recomenda-se que estas estejam majoritariamente vinculadas a maternidade onde será realizado o parto.

Durante o pré-natal os enfermeiros são responsáveis por um contato mais direto com as gestantes, criando um elo de confiança e respeito com as mesmas. Conforme predisposto pelo Ministério da Saúde (2007), o enfermeiro pode realizar o acompanhamento total da gestante de baixo risco, desde que esteja plenamente capacitado para atuar no pré-natal e no nascimento dos bebês, via conhecimentos teóricos e práticos previamente adquiridos. Contudo, muitas vezes a atuação dos enfermeiros no pré-natal é dificultada por limitações em sua formação, bem como na estrutura física e no processo de trabalho das Unidades de Saúde em que colaboram. Além disso, há um certo preconceito por parte das gestantes sobre o papel do enfermeiro ao longo do pré-natal, visto que, muitas não o reconhecem como profissional capaz de fornecer assistência adequada a gestação, sendo considerados apenas auxiliares do aparato médico.

Ao procurar assistência médica a gestante deve ser acolhida pelo enfermeiro, cabendo nesta etapa a confirmação da gravidez via realização do teste rápido, imunológico (TIG) ou Beta HCG. Após a confirmação da gestação pelo enfermeiro, a mãe deve ser cadastrada junto ao SUS, para a confecção de sua carteira de gestante. Concomitantemente, o enfermeiro deve iniciar um acompanhamento preventivo da gestante, via a anamnese, averiguando o histórico de saúde da paciente, as condições socioeconômicas, o nível de escolaridade, a idade, peso, pressão arterial, checagem no nível glicêmico, bem como a avaliação e atualização do esquema vacinal da paciente e a elaboração de um cronograma de consultas pré-natal.

Após as informações coletadas já na primeira consulta com o enfermeiro, a gestante deve ser classificada dentro da estratificação de risco em: Risco habitual, risco intermediário e alto risco. A partir da estratificação da gestante o enfermeiro deve orienta-la sobre os cuidados ao longo da gravidez, solicitar exames laboratoriais e de imagem, e quando necessário 
promover o encaminhamento para o amparo multiprofissional especializado (obstetra, cardiologista, neurologista, nutricionista, psicólogo, entre outros profissionais). Considerando ainda as informações elencadas pela anamnese, consultas, exames e a estratificação, o enfermeiro é responsável também por vincular a gestante ao hospital em que o parto deve ser realizado.

Ademais, o enfermeiro deve anotar na carteira da gestante todos os aspectos clínicos da paciente durante a gestação, tanto aqueles mais relevantes que sugerem maior risco, quanto os fatores preveníveis, mas que carecem de acompanhamento especializado. Assim, a estratificação de risco da gestante deve ser reavaliada durante as consultas do pré-natal, para que os riscos intercorrentes sejam rapidamente mitigados, reforçando a atenção das ações de assistência à saúde.

Os enfermeiros são responsáveis por ações importantes na assistência e promoção da saúde durante o pré-natal, mediando o acompanhamento das gestantes à medida que orientam essas e suas famílias sobre a vacinação, alimentação, amamentação, sintomas e fatores associados a riscos, como o uso de medicamentos, o consumo de álcool e drogas, prevenção de doenças endêmicas (dengue, zika vírus, febre amarela) e demais cuidados relacionados com a gestação e o puerpério.

Tais orientações devem ser realizadas pelo enfermeiro, preferencialmente o enfermeiro obstetra, por meio de palestras, reuniões em grupo, ou instruções individualizadas com as gestantes, as quais, devem acontecer periodicamente na unidade básica de saúde, e/ou nas consultas, e/ou nas visitas as gestantes, afim de que estas compreendam as modificações físicas e psicológicas decorrentes da gestação e sejam capazes de estabelecer ações que garantam a saúde e a qualidade de vida sua e do bebê.

Considerando a importância do enfermeiro no acompanhando pré-natal, bem como a diversidade de afazeres que este deve desempenhar ao longo de sua pratica profissional, questionamo-nos se "o enfermeiro, hoje, está atuando na assistência, na educação, na prevenção e na promoção do pré-natal de forma eficaz?’.

Por conseguinte, este trabalho teve como objetivo investigar por meio de uma revisão integrativa da literatura a atuação dos enfermeiros na educação e na promoção de saúde no pré-natal, avaliando os aspectos positivos e negativos desta interação, assim como suas limitações. A compreensão das limitações do atendimento pré-natal desempenhado pelo enfermeiro, possibilitou-nos orientar os mesmos ao longo desse estudo sobre ações que tendem a aprimorar o atendimento prestado no pré-natal. 


\section{Metodologia}

A pesquisa qualitativa foi realizada de forma descritiva, por meio de uma revisão integrativa da literatura nas bases de dados da Biblioteca Virtual em Saúde (BVS) e na SciELO (Eletronic Library Online) (Pereira, et al., 2019).

Foram considerados artigos científicos, teses e monografias, com pesquisas quantitativas, qualitativas e revisões bibliográficas que abordavam de forma clara o pré-natal e/ou o papel do enfermeiro no pré-natal. O material bibliográfico selecionado deveria conter título e resumo, serem escritos em português e publicados entre os anos de 2010-2020. Para a realização da pesquisa nestas bases de dados foram utilizadas as seguintes palavras chaves: enfermagem e o pré-natal, acompanhamento pré-natal e educação e promoção da saúde no pré-natal.

O material bibliográfico coletado foi submetido a dois momentos de classificação. No primeiro momento os artigos foram classificados considerando três critérios de inclusão: título, resumo e data de publicação. Posteriormente estes foram classificados segundo sua relevância perante o tema investigado, sendo lidos de forma criteriosa. Após a leitura foram considerados apenas artigos que respeitavam o objetivo proposto referente o papel do enfermeiro no pré-natal.

Os artigos aprovados pelos critérios de inclusão foram compilados de forma sistemática para compor os resultados no presente estudo, sendo seus pontos chaves organizados em tabelas no intuito de facilitar a discussão do material coletado. O número de fontes bibliográficas analisadas foi esquematizado na Tabela 1.

Tabela 1. Buscas nas bases de dados e categorização do material levantado.

\begin{tabular}{cccc}
\hline Base de dados & $\begin{array}{c}\text { Publicações } \\
\text { obtidas }\end{array}$ & $\begin{array}{c}\text { Publicações } \\
\text { excluídas }\end{array}$ & $\begin{array}{c}\text { Publicações } \\
\text { selecionadas }\end{array}$ \\
\hline Scielo & 360 & 353 & 7 \\
\hline BVS & 65 & 62 & 10 \\
\hline Total & 425 & 415 & 3 \\
\hline
\end{tabular}

Fonte: Os autores (2020). 


\section{Referencial Teórico}

\subsection{Um Breve Histórico do Pré-Natal}

Historicamente, a gestação representa um período de medos e incertezas para a mulher, contudo, apesar dos riscos a preocupação com a gestante e o bebê é recente, sendo proveniente de movimentos independentes e coletivos que aconteceram nos últimos dois séculos (Cruz, Caminha \& Batista-Filho, 2014).

Inicialmente os cuidados que envolviam o período gestacional eram restritos a alimentação, sem qualquer embasamento cientifico. Assim, durante a história evidenciamos o desamparo das mulheres no momento do parto, sendo auxiliadas apenas por outras mulheres, familiares, parteiras e/ou religiosas, as quais pautavam suas ações frente a experiencias anteriores (Coutinho, et al., 2003).

Considerando, tais limitações o aprimoramento do acompanhamento gestacional ao longo da história pode ser dividido em três fases. Inicialmente a assistência gravídica baseavase em orientações de cunho emocional e nutricional. Neste período foi implementado na França, o Centro de Proteção de Madame Becquet, criado por uma enfermeira, este tinha o intuito de acolher as gestantes em situação de vulnerabilidade social. Pinard, obstetra que atuava na instituição, inaugurou os estudos acerca dos prejuízos à gestante e ao feto frente a anormalidade fetal, indicando a necessidade de um acompanhamento frequente das alterações abdominais, instituindo assim a primeira menção ao que atualmente conhecemos por pré-natal (Cruz, Caminha \& Batista-Filho, 2014).

$\mathrm{O}$ início do século XX, foi marcado pela implementação da segunda fase do acompanhamento pré-natal nos Estados Unidos, quando o hospital Boston Lying-in-Hospital, começou a ofertar leitos de internação para gestantes e realizar visitas domiciliares. Na sequência (1910), a Itália implantou a primeira clínica de atendimento pré-natal, visando reduzir a mortalidade perinatal (Cruz, Caminha \& Batista-Filho, 2014). Enquanto isso no Brasil, o serviço de assistência pré-natal começou a ser ofertado apenas em 1925, por iniciativa do Professor Raul Briquet (Neme \& Zugaib, 2006).

A terceira fase do avanço da assistência pré-natal em meados do último século, foi acompanhada pela evolução da obstetrícia com a descoberta da ultrassonografia, favorecendo o acompanhamento da saúde da gestante e do feto (Cruz, Caminha \& Batista-Filho, 2014). 
Foi apenas neste período que a saúde da mulher foi reconhecida e considerada pelas políticas públicas de saúde no Brasil, contudo de forma ainda muito limitada perante as reais demandas e riscos da gestação, refletindo o machismo estrutural da época (Brasil, 2011).

Após a queda do regime ditatorial do Brasil em 1975, houve a emergência do movimento feminista, o qual lutava em prol do desenvolvimento e efetivação de políticas públicas voltadas a saúde da mulher, ao longo de todas as fases da vida (Gomes \& Sorj, 2014; Matuda, Aguiar \& Frazão, 2013). Fruto de diversas ações deste movimento foi instaurado em 1984 o Programa de Assistência Integral à Saúde da Mulher (PAISM) e o Programa de Assistência Integral à Saúde da Criança (PAISC). Estes atuaram alinhados as predisposições da Constituição Federal Brasileira, promulgada em 1988, e tinham o objetivo de diminuir a morbidade e mortalidade materna e infantil (Brasil, 2011).

Estimulado pelo estabelecimento da Constituição, o Sistema Único de Saúde (SUS) foi implementado em 1990, com a proposta de organizar, promover, proteger e recuperar a saúde de todos, conferindo acesso e assistência à saúde durante todas as fases da vida (Brasil, 2012). Porém, apenas em 2000 foi implementado pelo governo o Programa de Humanização no Pré-natal e Nascimento (PHPN), o qual tinha o intuito de acompanhar a gestante ao longo da gravidez, por meio de ações de promoção de saúde, prevenção e apoio psicossocial (Brasil, 2000).

As principais metas do PHPN visavam a redução das taxas de morbidade e mortalidade materna e perinatal, bem como ampliar a assistência dos profissionais de saúde ao longo da gestação, do parto, do puerpério e do período neonatal (Brasil, 2000).

Neste intuito, o PHPN regulamentou que durante o período gestacional a mulher deveria realizar no mínimo 6 consultas, uma no primeiro trimestre gravídico, duas no segundo trimestre, e três no último trimestre, as quais devem ser ofertadas pelo SUS (Brasil, 2000).

Desta forma, o Brasil destaca-se atualmente no cenário mundial pelo seu Sistema Único de Saúde (SUS), o qual realiza ações amplas de promoção da saúde, articuladas com políticas socioeconômicas no intuito de promover e garantir saúde as classes menos favorecidas (Silva, et al., 2016).

\subsection{Importância da Assistência Pré-Natal}

A $5^{\circ}$ meta do milênio estipulada pela Organização das Nações Unidas (ONU), consiste em ampliar o acesso à saúde sexual e reprodutiva, reduzindo a mortalidade materna, 
destacando o Pré-natal como uma ferramenta importante para o desenvolvimento humano (ONU, 2000).

Toda mulher adquire o direito ao acompanhamento pré-natal no momento em que engravida. O pré-natal representa um serviço importante de promoção e educação para a saúde da gestante, o acompanhamento realizado pelos profissionais da saúde neste período tem como objetivo atuar de forma preventiva na identificação de fatores de risco, afim de garantir o nascimento de uma criança saudável a medida que melhora a qualidade de vida da mãe e monitora o desenvolvimento do feto (Araújo, et al., 2010). Sintomas como hipertensão, diabetes e infecções, são facilmente identificados em exames de rotina realizados no pré-natal e reduzem consideravelmente a morbidade e mortalidade da mãe e o bebê (Viellas, et al., 2014; Silva, et al., 2016). Portanto, dificuldades na assistência pré-natal, tendem a desqualificar a saúde da gestante (Nunes, et al., 2016).

A Organização Mundial de Saúde (OMS), recomenda a realização de pelo menos 6 consultas, ao longo dos nove meses de gestação, as quais devem iniciar no primeiro trimestre e ter frequência mensal até a $28^{\circ}$ semana de gestação, seguidas de consultas quinzenais até o momento do parto (Duque, 2016).

Estudos atuais apontam que embora o pré-natal seja acessível em quase todo o Brasil, o inicio do acompanhamento dá-se de forma muito tardia, com apenas $73 \%$ das gravidas realizando as 6 consultas (Domingues, et al., 2012; Duque, 2016). O retardamento do início do acompanhamento, somado a falta de frequência nas consultas, prejudica a avaliação do desenvolvimento do feto, uma vez que dificulta o diagnóstico precoce de patologias gestacionais como diabetes e pré-eclampsia, fazendo com que problemas que seriam mitigados pelo acompanhamento adequado, causem problemas a saúde da mãe e do feto (Dias, 2014).

O Ministério da Saúde, ressalta que a qualidade da assistência pré-natal está relacionada a um cenário multifatorial, visto que para um bom funcionamento esta demanda de: profissionais qualificados, infraestrutura adequada, aparato instrumental mínimo, apoio laboratorial, material para registro, processamento e análise dos dados (Brasil, 2006).

A qualificação do acompanhamento pré-natal varia entre as regiões do Brasil, sendo afetada por fatores, como: a situação monetária e civil, a distância da residência, o estado de saúde, o nível de satisfação com o atendimento prestado, o tempo de duração da consulta e a demora no atendimento, a dificuldade na realização de exames e o acesso aos medicamentos (Serruya, Lago \& Cecatti, 2004). 
A contemplação de um aparato multifatorial adequado pelas Unidades Básicas de Saúde (UBS), tende a qualificar a maternidade, conferindo segurança as ações desempenhadas. Contudo, o elevado indicie de morbidade e mortalidade materna em algumas regiões ainda demanda atenção e a efetivação de uma assistência pré-natal mais qualificada (Araújo, et al., 2010; Vasconcelos-Moura, et al., 2014).

Embora a gestante seja o centro das ações desenvolvidas pelo pré-natal, é fundamental que haja o envolvimento da família ao longo de todo o processo, para que ambos sejam orientados, educados e assistidos neste momento complexo da vida, humanizando as interações e respeitando a mulher (Costa, et al., 2013).

A gravidez é um marco na vida da mulher e da família, sendo acompanhada por diversas alterações fisiológicas, decorrentes principalmente da flutuação hormonal e mecânica, as quais afetam todos os seus sistemas orgânicos, interferindo consideravelmente no sistema urinário e respiratório, no metabolismo, na deambulação e postura, e nos órgãos genitais. Assim, o pré-natal deve auxiliar a mulher a compreender tais alterações, educando-a sobre como portar-se diante destas, afim de que os riscos gestacionais sejam mitigados (Araújo, et al., 2010; Vasconcelos-Moura, et al., 2014).

A gestação reflete também nos aspectos psicológicos da gestante, gerando angustia, preocupação, medo e comoção. Assim, durante a gestação a mulher passa por uma reformulação na personalidade, desencadeada pelo alto estresse e por inseguranças, redefinido suas prioridades. Frente a estas alterações psicológicas, a mulher necessita de acompanhamento psicológico ao longo do pré-natal, bem como de uma assistência que a eduque sobre aspectos importantes da maternidade, como: nutrição, higiene, realização de atividades físicas regulares, atividade sexual, alterações corporais e planejamento familiar. Estas ações além de aprimorar a qualidade de vida da gestante devem orienta-la sobre os benefícios para sua saúde e a do bebê, instruindo-as sobre o parto normal e o aleitamento materno nos seis primeiros meses de vida. Portanto é fundamental que haja um contexto de apoio multidisciplinar para a gestante, afim de que esta crie vínculos de confiança com os profissionais que subsidiam a assistência pré-natal (Araújo, et al., 2010; Vasconcelos-Moura, et al., 2014).O acolhimento e amparo psicológico conferido pelo pré-natal, desde o primeiro contato até o puerpério, faz com que ao conhecer a gestante o profissional de saúde seja capaz de articular os serviços e a atenção necessária para cada caso, envolvendo os corpo multiprofissional da unidade de saúde, afim de fornecer a assistência adequada (Araújo, et al., 2010). 
Desta forma, para que o pré-natal seja ofertado de forma qualificada, as Unidades Básicas devem portar de boa estrutura física, para o atendimento da gestante, com salas privativas e bem ventiladas, acompanhadas por boas condições de higiene, além de ampla disponibilidade de recursos humanos, como: Médicos, enfermeiros, psicólogos e nutricionistas (Brasil, 2006).

Países desenvolvidos têm apresentado preocupação com a qualidade de vida da gestante ao longo do pré-natal, não restringindo-se apenas a ações de prevenção, manejo e detecção de problemas. Estes países tem buscado encorajar as mulheres frente aos aspectos psicossociais, compreendendo a complexidade familiar que permeia cada nova gestação (Castro, 2010).

Ao compreender os reflexos do pré-natal na melhoria da qualidade de vida da mulher, as ações desenvolvidas em prol da promoção da saúde, tendem a ser mais satisfatórias, visto que tornam factível o conhecimento acerca de quais dimensões da gestante estão sendo negligenciadas e demandam de aprimorando frente aquela realidade (Araújo, et al., 2010; Castro, 2010).

\subsection{Pré-Natal como uma Estratégia de Promoção de Saúde}

A promoção da saúde caracteriza-se por ser um processo de capacitação social para o aprimoramento da qualidade de vida e saúde da comunidade (Brasil, 2006). Assim, a efetivação de ações de promoção de saúde são compreendidas como ferramentas para o aprimoramento dos aspectos econômicos, sociais, político e culturais da sociedade (Castro, 2010).

Considerando que a promoção de saúde tende a melhorar diferentes dimensões da qualidade de vida, é responsabilidade do governo e dos setores da saúde, sociais e econômicos, atuar em prol de ações que visem a qualificação o itinerário de assistência do SUS (Brasil, 2006).

Uma vertente importante da assistência à saúde que deve ser melhorada e pensada de forma coletiva é o pré-natal, o qual, quando bem executado, reduz consideravelmente a vulnerabilidade social e os riscos à saúde condicionados a gestação. $\mathrm{O}$ acompanhamento prénatal deve nortear suas intervenções de promoção de saúde, alinhado a uma consciência de que a qualidade de vida materna é fruto de uma complexa produção social construída de maneira coletiva, e que deve ser considerada ao longo do contado com cada paciente (Castro, 2010; Durand \& Heidemann, 2013). 
As atividades desenvolvidas durante o pré-natal pelos profissionais da saúde detêm um cunho educativo, portanto, quando os profissionais da saúde se envolvem de maneira integral com as pacientes e seus familiares durante a assistência pré-natal, compreendendo suas dificuldades e carências, há um aprimoramento da promoção de saúde dos indivíduos e consequentemente na qualidade de vida destes (Souza, Roecker \& Marcon, 2010; Souza \& Fernandes, 2014).

A qualidade de vida da gestante deve ser considerada um produto da atuação efetiva do pré-natal. Nesta perspectiva, o pré-natal, deve preocupar-se em desenvolver atividades educativas junto as gestantes e suas famílias, orientando-as sobre aspectos relevantes do processo de desenvolvimento do feto, bem como das modificações corporais, emocionas e fisiológicas características do período (Souza, Roecker \& Marcon, 2010).

Ao longo do acompanhamento pré-natal os profissionais de saúde devem respeitar as questões culturais e religiosas de cada indivíduo, porém, concomitantemente, devem ser realizadas ações acerca do planejamento familiar, alimentação, rotina e amamentação, afim de promover a saúde da gestante antes e após o momento do parto (Duarte \& Almeida, 2014; Souza \& Fernandes, 2014).

Frente a este contexto é fundamental que os profissionais da saúde estabeleçam ações planejadas ao longo do pré-natal, que estejam amparadas em princípios que norteiem a promoção da saúde (Rocha, et al., 2011).

O ministério da Saúde destaca que o pré-natal é o melhor momento para que as ações de promoção de saúde relativas a maternidade sejam desempenhadas, assim, é factível que haja a realização de encontros que promovam o debate acerca de práticas saudáveis e a orientação sobre exercício que melhoram a qualidade de vida da gestante. Os quais, tendem a propiciar momentos de troca de experiencia entre as gestantes com os enfermeiros e/ou médicos, bem como entre as próprias gestantes, somando conceitos que melhoram a compreensão materna junto a gestação (Souza, Roecker \& Marcon, 2010; Dias, 2014).

O estabelecimento de estratégias de promoção da saúde deve considerar a singularidade de cada indivíduo, considerando-os como seres autônomos dotados de senso comum que devem ser direcionados pelos profissionais da saúde a desenvolverem-se por meio de atividades que promovam o aprimoramento da saúde materna e reflitam em melhorias na qualidade de vida (ROCHA et al, 2011).

Os profissionais da saúde devem agir de forma articulada com um processo de transformação permanente, o qual deve ser capaz de reconhecer a vulnerabilidade e os riscos de doença da gestante, auxiliando no encaminhamento adequado junto a uma equipe 
multiprofissional, agindo preventivamente afim de mitigar os problemas provenientes da gestação (Castro, 2010; Rocha, et al., 2011; Durand \& Heidemann, 2013).

A ineficiência do acompanhamento e assistência pré-natal, promovem aumento nas taxas de mortalidade e morbidade materna e do feto, aumentando a prematuridade, e o baixo desenvolvimento infantil. Desta forma, o pré-natal alinhado aos preceitos de promoção da saúde, promove uma melhora na qualidade de vida da mãe e do feto, visto que viabiliza a intervenção oportuna dos profissionais da saúde em momentos decisivos da gestação (Cruz, Caminha \& Batista-Filho, 2014).

A promoção de estratégias de saúde as usuárias do pré-natal, pautam-se em políticas públicas que tem o intuito de ofertar a toda a população uma assistência qualificada a mãe e ao feto, acompanhando todas as etapas do desenvolvimento gestacional. Contudo, ainda existem limitações no processo de promoção de saúde pelo pré-natal nas diferentes regiões no país, as quais são potencializadas pela falta de padronização nos registros de acompanhamento, dificultando a fiscalização pelo poder público (Duque, 2016).

Visando melhorias na assistência pré-natal em todo o território nacional, foi implementado uma ficha de acompanhamento da gestante, denominado SispreNatal, este consiste em um software ligado a base de dados do DATASUS, para controlar os atendimentos prestados em todo o território nacional, forçando os municípios a realizarem o atendimento adequando da gestante sob pena de corte de recursos (Brasil, 2000; Brasil, 2014).

Tal ferramenta aumenta a fiscalização sobre as ações de promoção de saúde desenvolvidas no pré-natal nos diferentes municípios do Brasil. Auxiliando na promoção de uma maternidade segura, amparada por estratégias de humanização dos cuidados com a saúde nas mais afastadas regiões do país, respeitando os princípios de integridade e universalidade da atenção à saúde (Brasil, 2014; Cruz, Caminha \& Batista-Filho, 2014).

O envolvimento das classes marginalizadas da sociedade, promove a diminuição das iniquidades relacionadas a saúde materna. Considerando esta perspectiva, os profissionais da saúde devem agir de forma acolhedora, compreendendo a diversidade humana e orientando ações que promovam qualidade de vida da gestante e do ser que está sendo gerado, tanto junto ao atendimento corriqueiro, quanto a assistência hospitalar de alto risco (Brasil, 2005; Fernandes, 2016). 


\subsection{Atuação do Enfermeiro no Acompanhamento Pré-Natal}

Dente os profissionais capacitados para a realização do pré-natal, destacamos que a assistência pode ser desempenhada por um médico obstetra, mas também por outros profissionais da saúde, como enfermeiros e enfermeiros obstetras. Segundo a Lei 94.406/87, o enfermeiro é apto a realizar o pré-natal de baixo risco, podendo fazer consultas, prescrever medicamentos, assistir e educar a mulher quanto a aspectos relevantes a sua saúde (Araújo, et al., 2010).

Desta forma, o enfermeiro assume uma posição importante ao longo do acompanhamento pré-natal, sendo preparado para atender a gestante e atuar em prol de ações de promoção e prevenção da saúde, educando-a e esclarecendo dúvidas acerca de aspectos relevantes a manutenção da saúde (Martins, et al., 2015).

Durante a assistência pré-natal o enfermeiro deve compreender a singularidade da mulher, examinando e investigando de forma criteriosa aspectos que podem indicar situações de risco para a gestação, e/ou que podem limitar o desenvolvimento do embrião (Martins, et al., 2015; Duque, 2016).

Os enfermeiros dever atuar em harmonia com os demais profissionais da saúde que compõem o atendimento pré-natal, afim de que o atendimento seja executado de maneira integral e satisfatória, expondo as demandas provenientes de cada paciente (Martins, et al., 2015; Silva, 2018).

Ademais, é fundamental que seja estabelecida uma comunicação sincera entre a gestante e o enfermeiro, a qual deve acontecer de maneira fluida, sem pré-conceitos e julgamentos, criando um vínculo de confiança entre a mãe e o profissional enfermeiro. Facilitando a identificação dos fatores de risco, bem como a detecção de pontos mais importantes para a educação da saúde de cada mulher (Martins, et al., 2015).

O contato direto com a gestante amplia a concepção dos profissionais enfermeiros acerca das ideias, opiniões, pensamentos, medos e inquietações da mulher. Assim, o diálogo e a posição de ouvinte do enfermeiro junto a gestante, humaniza o atendimento pré-natal, à medida que confere confiança junto ao aparato de saúde, fortalecendo o percurso da gestante ao longo do pré-natal, até ao parto (Campos, et al., 2016; Silva, 2018).

Portanto, é fundamental que os enfermeiros compreendam a necessidade de voltarem sua ação não apenas aos conceitos biológicos, mas também as características emocionais, mecânicas, socioeconômicas, culturais e psicossociais da gestante. Ao considerar tal 
complexidade o enfermeiro aprimora a atenção prestada a gestante, garantindo uma melhora na saúde do binômio mãe e bebê (Rodrigues, 2015).

\subsection{A Consulta da Enfermagem e as Competências Clinicas do Enfermeiro no Acompanhamento Pré-Natal}

A consulta pré-natal representa um momento singular da vida da mulher, em que esta acessa o Sistema de Saúde e abre uma brecha para que ações de prevenção, promoção e recuperação da saúde. Tais ações possuem cunho multidisciplinar com diversos profissionais vinculados as Unidades Básicas de Saúde, neste contexto o enfermeiro detém papel central, coordenando e supervisionando as intervenções que devem ser realizadas (Rodrigues, 2015; Rios \& Vieira, 2007).

Durante a realização da primeira consulta do pré-natal o enfermeiro deve realizar uma série de procedimentos junto a gestante, os quais são simples, porém fundamentais para a qualidade de vida ao longo da gestação. Assim, o enfermeiro deve agir alinhado com o plano de acompanhamento da gravidez, pautando suas ações frente a cada semana gestacional (Viellas, et al., 2014; Martins, et al., 2015; Silva, 2018).

Neste momento o enfermeiro deve ouvir a gestante, realizando o levantamento de dados via anamnese, estabelecendo um vínculo de confiança. A anamnese deve ser conduzida com o intuito de verificar o histórico clínico da mulher, bem como seu histórico obstétrico e familiar. Todas as respostas devem ser consideradas, afim de que a singularidade de cada mulher e/ou gestação possa ser compreendida. Ademais, frente as informações coletadas o enfermeiro deve orientar e educar a mulher sobre pontos importantes para assegurar sua saúde e solicitar exames gerais e obstétricos para avaliação da gestação em curso (Rodrigues, 2015; Silva, 2018).

Durante as consultas com o profissional enfermeiro o prontuário da gestante deve ser preenchido de forma criteriosa, sendo notadas informações como idade, peso, estatura, altura uterina, pressão arterial, aspectos das mamas, batimentos cardíacos do feto, antecedentes de risco. Tais informações devem ser vinculadas ao cartão da gestante e consideradas para a estratificação de risco, sendo revisadas pelo enfermeiro a cada consulta do pré-natal (Martins, et al., 2015; Duque, 2016).

Considerando os aspectos clínicos da gestante e a presença de fatores de risco relacionados a morbidade e mortalidade materna e infantil, o binômio mãe-criança pode ser ranqueado em três estratificações de risco pelo enfermeiro: 1- Risco habitual; 2- Risco 
intermediário; e 3- Alto risco (Paraná, 2017).

As gestantes classificadas como de risco habitual, são aquelas que não possuem: comorbidades, vulnerabilidade sociodemográfica e histórico predisponente a risco. Enquanto que aquelas classificadas como de risco intermediário, apresentam riscos individuais, como: baixa escolaridade, idade acima de 40 anos, etnia (negra e indígenas), ocorrência de aborto, natimorto ou óbito em gestações anteriores. Já as gestações estratificadas como de alto risco estão relacionadas a presença de comorbidades pré-existentes e/ou intercorrências clinicas relacionadas a gestação, como hipertensão gestacional, má formação fetal, baixo desenvolvimento uterino, sangramento, entre outros (Paraná, 2017).

A gestante deve ser informada sobre os tipos e a escolha do parto recomendado frente a sua estratificação de risco e também quanto aos cuidados ao longo da gestação e do puerpério, afim de conduzir uma gestação saudável e equilibrada (Viellas, et al., 2014; Silva, 2018).

A realização correta da anamnese auxilia no estabelecimento adequado da estratificação de risco e possibilita a vinculação adequada ao pré-natal, norteando a escolha do melhor local para que as demandas de cada gestação sejam compreendidas. Assim, a estratificação correta diminui consideravelmente a mortalidade materna e infantil e melhora a qualidade de vida durante a gestação e no puerpério (Paraná, 2017).

$\mathrm{O}$ enfermeiro destaca-se neste período como um elo importante entre a gestante a o sistema de saúde. Este atua na prevenção e promoção da saúde da mãe, estabelecendo ações educativas que auxiliam na compreensão das alterações físicas, fisiológicas e comportamentais relativas a este período, bem como a cuidados que a mãe e a família devem adotar durante e após a gestação, como: alimentação, amamentação, vacinação e ações de higiene básica. Ademais, a humanização do cuidado promovido pelo profissional de enfermagem em seu cotidiano com a gestante deve criar um ambiente harmonioso e de confiança, no qual a gestante sinta-se segura e acolhida (Martins, et al., 2015; Nunes, et al., 2016).

Ademais, durante as consultas cabe ao enfermeiro orientar a gestante sobre o trabalho de parto, as dores do parto e os riscos e comportamentos durante o parto e puerpério. A mulher deve conhecer os procedimentos do parto, afim que de que esteja consciente sobre o que é ou não, recomendado para a sua situação, bem como o tempo que as alterações fisiológicas levam para a viabilização do parto. Ao compreender tais variáveis, a gestante ganha autonomia, policiando ações desrespeitosas e precipitadas por parte dos profissionais da saúde, agindo em prol da naturalização e humanização do parto (Viellas, et al., 2014; 
Duque, 2016).

Aproveitando-se do elo estabelecido com a gestante o enfermeiro deve instigar a participação e o envolvimento de algum membro da família da gestante durante o pré-natal, visto que por conceber um convívio maior com a gestante o familiar tende a exteriorar informações importantes, que auxiliam na compressão da singularidade da saúde daquela mulher (Cabral, Hirt \& Van Der Sand, 2013; Holanda, et al., 2018).

Assim, para a gestante que possui um parceiro, o pré-natal caracteriza-se como um momento propicio para que o casal seja instigado a debater sobre a organização e o planejamento familiar, debatendo sobre a pretensão futura de ter ou não outros filhos e sobre quais são os melhores métodos contraceptivos dentro desta perspectiva, fazendo com que esta decisão não fique restrita a mulher (Duque, 2016; Holanda, et al., 2018).

Ao envolver os parceiros com o pré-natal, o enfermeiro pode perceber também problemas relacionados a convivência do casal, que podem atribuir riscos a integridade física da mulher e da criança. Ademais, a gestante vítima de agressão domestica tende a adiar o início do acompanhamento pré-natal por medo. Tais pacientes geralmente apresentam uma ocorrência maior de depressão, hemorragias, partos prematuros e abortos espontâneos (Durand \& Heidemann, 2013; Rodrigues, et al., 2014; Holanda, et al., 2018).

Os enfermeiros devem estar a par das condições socioeconômicas da comunidade onde trabalham, visto que, estas estão intrinsicamente relacionadas com a vulnerabilidade a violência e a dependência de drogas licitas e ilícitas. Além de tal contexto favorecer a ocorrência de agressão, a gestantes detêm maior probabilidade de ser usuária de algum tipo de entorpecente, fator que expõe mãe e criança a diversos riscos e demanda maior atenção e senso crítico do enfermeiro durante a anamnese e a assistência pré-natal (Kassada, Marcon \& Waidman, 2014; Duque, 2016).

Ao detectar que a gestante está exposta a alguns desses fatores de risco, o enfermeiro deve buscar auxílio de um aparato profissional especializado para o atendimento de intercorrências desse tipo. $\mathrm{O}$ aparato multiprofissional acionado, demanda de ações planejadas e assertivas, com vista a resgatar a gestante de uma condição de risco e reestabelecer a saúde a mãe e ao neonato (Rodrigues, et al., 2015; Duque, 2016).

Assim, a valorização de diferentes atores no pré-natal, confere maior humanização ao longo da assistência à saúde, estimulando a autonomia da mulher e fortalecendo os vínculos de confiança da sociedade para com o Sistema de saúde (Durand \& Heidemann, 2013; Silva, 2018).

Porém os cuidados da enfermagem não devem ficar restritos apenas ao 
acompanhamento da gestação, devendo ser prolongados após o parto, para que haja a qualificação pela da saúde da mulher (Silva, 2018).

O puerpério é caracterizado como o período pós-parto, sendo dividido em três momentos, o primeiro chamado de puerpério imediato, estende-se até o decimo dia, seguido do puerpério tardio até o $42^{\circ}$ dia, e o puerpério remoto até um ano após o parto. O puerpério representa o momento de maior risco e vulnerabilidade materna, sendo atribuído a este período o maior índice de morbidade e mortalidade neonatal e materna, as quais são frequentemente decorrentes de alterações arteriais severas, hemorragias, infecções e abortos (Souza \& Fernandes, 2014).

Em contrapartida, neste período a assistência pré-natal sofre uma ruptura, e a mãe fica desassistida em seu momento de maior vulnerabilidade, física, emocional e psicológica (Souza \& Fernandes, 2014). Assim, é sugestível que ao longo do período puerperal o enfermeiro realize visitas e consultas a mãe e ao bebê, para que as demandas e as carências pertinentes a esse período sejam supridas via um profissional capacitado. O enfermeiro deve assim, reafirmar as instruções acerca do aleitamento materno, além disso, neste período devem acorrem orientações sobre a retomada do ciclo menstrual e da atividade sexual, educando a mãe sobre a possíveis dificuldades e os sinais que merecem atenção (Brasil, 2006).

Além disso, a gestante precisa receber instruções sobre a importância da amamentação para o desenvolvimento do bebê, bem como sobre os cuidados com as mamas e mamilos durante o aleitamento, considerando o tipo de mamilo de cada mulher para a realização das orientações (Duque, 2016; Rodrigues, 2015; Rios \& Vieira, 2007).

Considerando o predisposto na legislação (lei 7.498 de 25 de julho de 1986) sobre a atuação do profissional de enfermagem, este possui embasamento teórico e prático para o acompanhamento pré-natal de gestantes de baixo risco de maneira intercalada com o médico, estando apto para realizar consultas e intervir de forma efetiva e qualificada na promoção e no aperfeiçoamento da qualidade de vida da gestante (Silva, et al., 2016). Cabe ao enfermeiro planejar suas ações conforme a necessidade de cada indivíduo, para que questões educativas como autocuidado, alimentação e fatores de risco para a saúde da gestante sejam discutidos durante o pré-natal (Costa, et al., 2013).

Além disso, o enfermeiro está apto para prescrever medicações e solicitar exames, realizando encaminhamentos a atenções de saúde secundárias e terciarias, considerando a estratificação de risco vinculada a gestante (Nunes, et al., 2016).

Por outro lado, notasse que a atividade do enfermeiro no pré-natal ainda não é 
realizada efetivamente conforme o disposto pela legislação e pelas normas no Ministério da Saúde, devido à resistência das instituições em romper o modelo médico clássico. As consultas com o enfermeiro acontecem apenas quando a gestante não tem acesso ao atendimento obstétrico, dificultando o reconhecimento do enfermeiro como profissional capacitado para o atendimento pré-natal (Viellas, et al., 2014; Martins, et al., 2015; Silva, et al., 2016).

Ademais, a assistência prestada pelos enfermeiros durante o pré-natal é frequentemente prejudicada devido à baixa estrutura física, a precariedade e falta de materiais adequados, prejudicando a qualidade do atendimento. Visto que a realização de consultas em locais adequados está relacionada com a segurança e privacidade da gestante e do enfermeiro, criando um ambiente de empatia e confiança, onde medos e dúvidas podem ser trabalhados, contribuindo consideravelmente com a educação e promoção de saúde entre os pares (Martins, et al., 2015; Silva, et al., 2016).

Outro fator de prejudica atuação do enfermeiro no pré-natal é a precariedade de recursos humanos do sistema público de saúde, sobrecarregando os profissionais ativos com o acúmulo de funções e alto número de atendimento diários. Aspectos que refletem em atendimentos superficiais e pouco eficazes na identificação de patologias associadas a gestação (Costa, et al., 2013; Viellas, et al., 2014; Campos, et al., 2016; Silva, et al., 2016).

Além disso, a sobrecarga tem promovido, em partes, a baixa qualificação profissional, visto a ausência de estímulos para a capacitação e aperfeiçoamento continuo destes profissionais, bem com a falta de instruções para a adoção de protocolos atualizados para aprimorar e sistematizar a assistência pré-natal (Rocha, et al., 2011; Queiroz, et al., 2015; Silva, et al., 2016).

Torna-se evidente a necessidade de orientação e educação dos enfermeiros, acerca do acompanhamento pré-natal. Assim, para o aprimoramento da qualidade do atendimento prestado a gestante é recomendada a adoção de protocolos norteadores, que discriminem passo-a-passo as ações que o enfermeiro deve realizar durante a consulta pré-natal, objetivando a qualificação do serviço prestado e garantindo maior eficácia no atendimento (Costa, Marinho \& Moraes, 2015).

Tais limitações fazem com que muitas mulheres tenham uma visão equivocada do papel do enfermeiro durante o acompanhamento pré-natal, percebendo-o como um veículo de assistência técnica ao aparato médico e desconhecendo seu direito a consulta com enfermeiros. Apontando assim, para a ausência de reconhecimento dos profissionais da enfermagem como atores centrais na assistência pré-natal (Silva et al, 2016). 
Desta forma, recomenda-se a implementação de fichas clínicas bem organizadas e ricas em informações sobre a mãe e a criança. Parte dessas fichas, comtempla apenas questões referentes a gestação, contudo excluem o período do parto, o puerpério e a saúde do recémnascido. Ao comtemplar todos esses estágios o enfermeiro assegura maior abrangência no atendimento pré-natal, tornando as intervenções mais assertivas (Duarte \& Mamede, 2013).

Considerando, a realização nacional do pré-natal, observamos discrepâncias no atendimento ofertado pelos enfermeiros durante as consultas. Tais variações, decorrem da ausência de um instrumento unificado, embasado em instrumentos científicos e de aplicação nacional, os quais, otimizariam as informações acerca da gestante e sistematizariam as ações de prevenção e promoção da saúde (Duarte \& Mamede, 2013).

Embora a cobertura pré-natal no Brasil apresente-se de forma homogênea, alguns fatores, além da capacitação profissional, destacam-se, visto que grande parte das gestantes tem acesso tardio ao atendimento, realizando um número baixo de consultas devido à falta de profissionais e a baixa infraestrutura, resultando uma identificação tardia dos problemas e conferindo riscos à saúde da gestante e do bebê (Costa, et al., 2013; Viellas, et al., 2014).

Portanto, é crucial que a relação dos enfermeiros com a promoção e educação para saúde durante o pré-natal seja investigada de forma extensiva, para traçar caminhos no intuito de sanar as lacunas presentes ao longo desse processo.

\section{Resultados e Discussão}

Os enfermeiros destacam-se como atores fundamentais para a qualificação e efetivação plena do pré-natal, desempenhando ações de promoção, prevenção e assistência à saúde das gestantes e dos neonatos. Portanto, é crucial que compreendamos os aspectos positivos e negativos desta interação, afim de que compreendendo-os possamos traçar estratégias de aprimoramento.

Foram encontradas 425 publicações em ambas as bases de dados (Scielo e BVS), destas 415 não se enquadraram nos critérios pré-estabelecidos para o desenvolvimento da pesquisa, sendo selecionados um total de 10 publicações, que contribuíram consideravelmente para com a compreensão do papel do enfermeiro no acompanhamento pré-natal, dados apresentados na Tabela 1.

Lima e Corrêa (2012), destacam que o desempenho das atividades relativas ao SISPRENATAL, bem como preenchimento da ficha são majoritariamente executados pelo profissional enfermeiro. Neste momento informações da gestante consideradas "não- 
obrigatórias" pelo sistema digital tendem a ser negligenciadas, tais como: endereço, raça e cor, fatores importantes que podem indicar ao enfermeiro a condição socioeconômica da gestante, movimentando sua classificação na estratificação de risco, conforme ressaltam o material bibliográfico sistematizado na Tabela 2 (Paraná, 2017).

Tal incoerência no preenchimento do sistema indica que os enfermeiros carecem de aprofundamento teórico para o gerenciamento satisfatório do SISPRENATAL, incorporando junto as fichas todas as nuances da gestante (Lima \& Corrêa, 2012).

Tabela 2. Aspectos relevantes do material bibliográfico selecionado.

\begin{tabular}{|c|c|c|c|}
\hline $\begin{array}{l}\text { Autoria e } \\
\text { ano }\end{array}$ & Título & Objetivos & $\begin{array}{c}\text { Principais } \\
\text { resultados/conclusões }\end{array}$ \\
\hline $\begin{array}{l}\text { Corrêa, } \\
2012 .\end{array}$ & $\begin{array}{c}\text { A produção de dados } \\
\text { para o Sistema de } \\
\text { Informação do Pré-Natal } \\
\text { em unidades } \\
\text { básicas de saúde. }\end{array}$ & $\begin{array}{l}\text { Esta pesquisa objetivou } \\
\text { analisar o processo de } \\
\text { produção de dados para o } \\
\text { SISPRENATAL em } \\
\text { Unidades Básicas de Saúde } \\
\text { de Cuiabá, MT, Brasil. }\end{array}$ & $\begin{array}{c}\text { A análise dos dados revelou } \\
\text { inexistência de definição da } \\
\text { participação da equipe na } \\
\text { produção dos dados e modos } \\
\text { diversos de preenchimento das } \\
\text { fichas do sistema. O } \\
\text { conhecimento dos profissionais } \\
\text { sobre muitos aspectos das fichas } \\
\text { foi divergente, o preenchimento } \\
\text { das fichas foi feito de modo } \\
\text { inadequado e foram verificadas } \\
\text { falhas no sistema informalizado. } \\
\text { Medidas como a capacitação dos } \\
\text { profissionais, a revisão do } \\
\text { sistema e de suas fichas são } \\
\text { indispensáveis para a produção } \\
\text { de informações fidedignas sobre } \\
\text { a assistência pré-natal no } \\
\text { município. }\end{array}$ \\
\hline $\begin{array}{l}\text { Lima, } \\
2013 .\end{array}$ & $\begin{array}{c}\text { Enfermagem no pré- } \\
\text { natal de baixo risco na } \\
\text { estratégia }\end{array}$ & $\begin{array}{l}\text { Descrever a experiência das } \\
\text { mulheres grávidas no } \\
\text { atendimento pré-natal e de } \\
\text { baixo risco na consulta de }\end{array}$ & $\begin{array}{l}\text { As vivências com a equipe e com } \\
\text { a população ampliaram a visão } \\
\text { sobre a importância do } \\
\text { enfermeiro, no que se refere à }\end{array}$ \\
\hline
\end{tabular}


Saúde da Família.

enfermagem, residentes na área de cobertura da

Estratégia de Saúde da

Família, em uma cidade do

Rio Grande do Sul (Brasil),

por meio da interação e do

fortalecimento do vínculo

das mulheres gestantes com

o serviço.
Alves, et Cuidado pré-natal e

al., 2015. cultura: uma interface na atuação da enfermagem
Conhecer as práticas de

cuidado e os valores

culturais de enfermeiras ao assistir à gestante gerência dos serviços de saúde e à promoção de ações educativas e de cuidado da saúde das mulheres no ciclo gravídico puerperal.
O cuidado de enfermagem transcendeu condutas tecnicistas e que sofreu influência de fatores culturais da percepção individual de cada enfermeira. Promover o cuidado permeado pela cultura é uma interface necessária na enfermagem, uma vez que por meio do reconhecimento do contexto sociocultural da gestação se identificam os caminhos para que o cuidado culturalmente congruente seja realizado.

\section{O atendimento da enfermeira é} diferenciado, com acolhimento e

Descrever a percepção das

Spindola,

Progianti

\& Penna,

2012.

Opinião das gestantes sobre acompanhamento da enfermeira obstetra no pré-natal de um hospital universitário gestantes em relação ao atendimento

da enfermeira obstetra no acompanhamento do prénatal. atenção, facilitando o

esclarecimento de dúvidas e o aprendizado em relação ao processo de gestar e parir. $\mathrm{O}$ vínculo estabelecido entre a enfermeira obstetra e a gestante no pré-natal contribui para o processo de educação para a saúde das mulheres, respeitando- 
se os hábitos de vida e a cultura da clientela

O estudo apontou fragilidades no que tange à integralidade, humanização,

acolhimento, ausência de vínculo, uso indiscriminado de tecnologias e intervenções desnecessárias, as quais podem produzir e/ou potencializar

Atendimento pré-natal

Cabral,

Hirt \& puérperas: da

Sand, medicalização à

2013.

$$
\begin{aligned}
& \text { fragmentação do } \\
& \text { cuidado. }
\end{aligned}
$$

\section{Conhecer a percepção de puérperas sobre}

atendimento em serviço de pré-natal. situações de vulnerabilidade. Os resultados sinalizam a necessidade de reorganização da atenção no pré-natal e nascimento, sob a lógica da longitudinalidade do cuidado, tanto nos serviços públicos como privados e de pactuação de ações intersetoriais nos modos de promoção da saúde das mulheres e de fomento à formulação de políticas públicas mais equânimes e positivas na perspectiva da integralidade da atenção.

\begin{tabular}{cc}
\hline & \\
& $\begin{array}{c}\text { Amamentação: a prática } \\
\text { do enfermeiro na }\end{array}$ \\
Chaves, & perspectiva da \\
et al., & Classificação \\
2011. & Internacional de \\
& Práticas de Enfermagem \\
& em Saúde Coletiva.
\end{tabular}

Descrever os diagnósticos e as intervenções de Enfermagem sob a perspectiva da

Classificação Internacional das Práticas de Enfermagem em Saúde Coletiva - CIPESC ${ }^{\circledR}$ na atenção à Saúde da Mulher, subtema Pré-Natal
O diagnóstico Amamentação

Adequada foi o mais frequente e as intervenções mais acionadas relacionam-se ao fortalecimento da usuária frente ao processo saúde-doença $(68,9 \%)$. Apesar da atuação do Enfermeiro no puerpério, esta competência não consta do Protocolo do Programa. Concluiu-se que são 
e Puerpério,

correlacionando-os com as competências do

Enfermeiro no Programa

Mãe Curitibana. necessários pequenos ajustes nos diagnósticos analisados e uma revisão do Protocolo para abrigar as competências do Enfermeiro que são desenvolvidas em sua prática nos serviços de saúde, conforme constam nos registros da base CIPES.

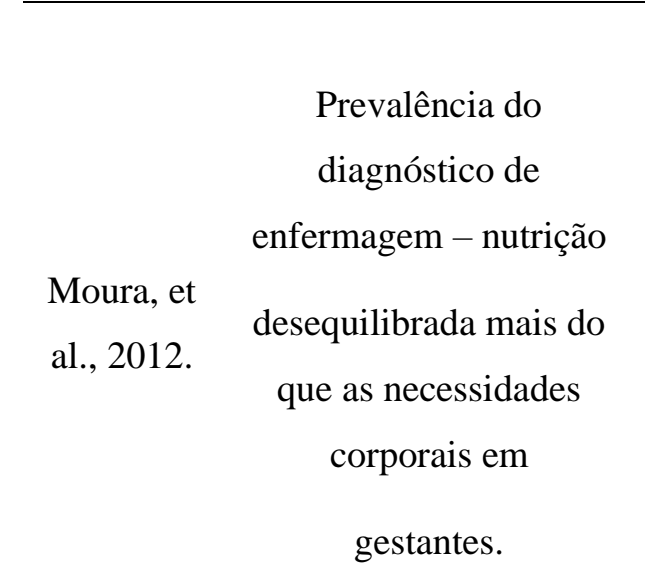

Barbieri, Análise da atenção préet al., natal na percepção de $2012 . \quad$ puérperas.
Identificar a prevalência do

$$
\text { Diagnóstico de }
$$

Enfermagem (DE) nutrição desequilibrada: mais do que as necessidades corporais em gestantes e verificar a associação entre o DE e as variáveis

sociodemográficas e obstétricas.
O DE pesquisado apresentou prevalência marcante nas gestantes pesquisadas, estando associado a fatores evitáveis por ações educativas voltadas ao planejamento familiar, como idade materna e paridade.
Analisar a atenção pré-natal na percepção de usuárias que realizaram o parto em um hospital universitário, de uma cidade de médio porte do RS e caracterizar as práticas de pré-natal ofertadas neste município.
Apesar do alto índice de realização de pré-natal, uma análise apurada evidencia uma desarticulação entre o período pré-natal, parto e puerpério e revela a importância de uma abordagem interdisciplinar no pré-natal a exemplo da praticada pelo Programa de Residência multiprofissional em que o estudo foi realizado.

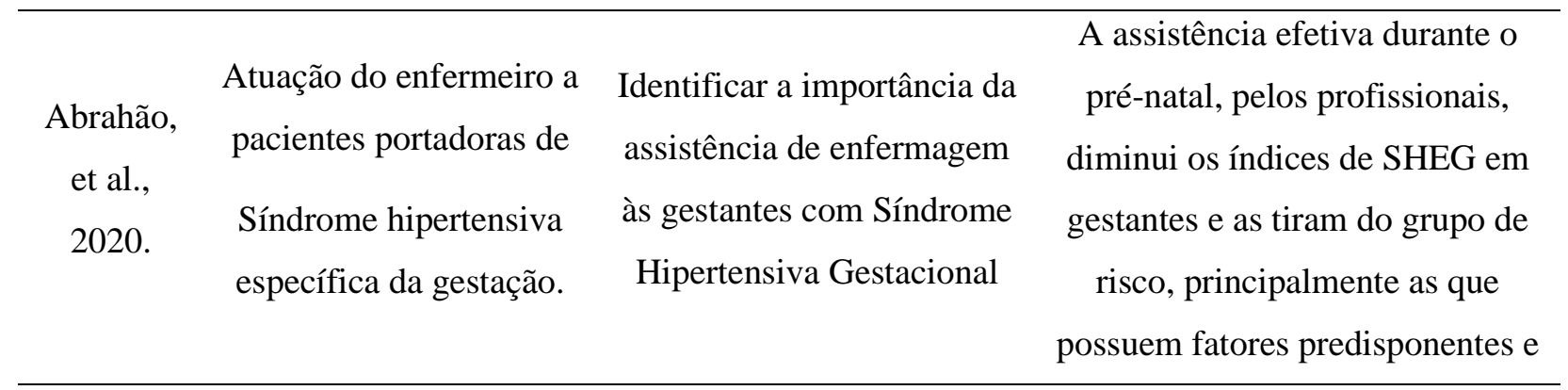


etiológicos. Assim, é possível

descrever a atuação do

enfermeiro frente à patologia, observando a necessidade de um trabalho conjunto à uma equipe multidisciplinar para que ocorra um atendimento eficaz à gestante.

Considera-se relevante a

$\begin{array}{ccc} & \text { Consulta de } & \text { Realizar uma revisão } \\ \text { Santos, } & \frac{\text { Enfermagem no pré- }}{\text { natal de baixo risco: }} & \text { bibliográfica na literatura } \\ \text { 2011. } & \frac{\text { Gestograma de rotinas }}{\text { básicas. }} & \text { natal, comal sobre o tema pré- } \\ & & \begin{array}{c}\text { subsidiar umalidade de } \\ \text { educativa em serviço. }\end{array}\end{array}$
educação permanente em saúde para que os trabalhadores possam ser capacitados, aprimorando sua atuação e permitindo uma maior valorização da consulta de enfermagem no pré-natal de forma correta e não somente como um atendimento de rotina.

Fonte: Autores (2020).

Cabral, Hirt e Sand (2013), relataram em seu estudo a presença de fragilidades na integridade da assistência pré-natal, com baixa articulação entre os agentes de saúde, e o uso indevido de tecnologias, refletindo em limitações no acolhimento e no estabelecimento de vínculos. Assim, estes orientam para a necessidade de reorganização nas ações de promoção de vínculos para o aprimoramento do acompanhamento pré-natal (Tabela 2).

Ademais, os enfermeiros enfrentam ainda dificuldades no acompanhamento pré-natal, devido à falta de reconhecimento do seu papel junto à comunidade, demandando de esforço extensivo para o estabelecimento de vínculo com a gestante. Desta forma, os enfermeiros devem insistir na formação de grupos de debates com as gestantes sobre aspectos relevantes a gestação e o puerpério, estabelecendo frequência nas consultas de enfermagem. Ao fortalecer o elo da enfermagem com o pré-natal, os enfermeiros promovem maior autonomia a gestante e aos profissionais da saúde, qualificando a assistência prestada (Lima, 2013; Spindola, Progianti \& Penna, 2012).

Em contrapartida, outros trabalhos indicam uma atuação satisfatórias dos enfermeiros na assistência pré-natal, afirmando que os enfermeiros tem ouvido atentamente as gestantes, 
considerando suas dificuldades e angústias, afim de promover ações de cunho humanizado que estimulam a continuidade das consultas e a efetivação de um pré-natal de qualidade (Spindola, Progianti \& Penna, 2012; Alves, et al., 2015). Alves e colaboradores (2015), destacam que o acompanhamento dos enfermeiros tem transpassado as intervenções tecnicistas, envolvendo fatores culturais pessoais, os quais, agregam maior carisma junto as gestantes e projetam caminhos para uma assistência satisfatória.

Ademais, o vínculo da consulta pré-natal é fortalecido quando os enfermeiros respeitam as crenças e valores intrínsecos de cada mulher, considerando tais singularidades para o esclarecimento de dúvidas e para as orientações acerca da alimentação, hábitos de vida, amamentação e cuidados pessoais e com o bebê (Chaves, et al., 2011; Spindola, Progianti \& Penna, 2012). Portanto, para atuar de forma plena na assistência pré-natal, os enfermeiros tem norteado sua atividade considerando tanto os fatores fisiológicos, quanto socioculturais da gestante (Alves, et al., 2015).

A adoção de um vínculo integrativo ao longo das consultas da enfermagem, age como um aliado para a continuidade da assistência pré-natal, sendo também fator relevante na escolha da unidade de saúde por outras mulheres, as quais consideram os relatos positivos de familiares que passaram pela unidade (Spindola, Progianti \& Penna, 2012).

Barbieri e colaboradores (2012), evidenciaram que as puérperas estavam satisfeitas quanto a educação ofertada no pré-natal acerca de aspectos como amamentação, contracepção, cuidados com o bebê e com a alimentação. Por outro lado, Moura e colaboradores (2012), relatam que cerca de 68\% das gestantes possuem desbalanço nutricional, com a ingestão de nutrientes acima das demandas metabólicas. Desta forma, a promoção de saúde pelos enfermeiros ao longo do pré-natal, é crucial para o distanciamento de problemas como o sobrepeso e a má alimentação no período gestacional.

As consultas da enfermagem devem acompanhar o estado nutricional da gestante, orientando-a e educando-a acerca dos aspectos relevantes as necessidades do seu corpo. Assim o enfermeiro obstetra deve utilizar o contato próximo com a gestante como uma ferramenta de transformação dos hábitos alimentares e motores, articulando atividades de instiguem e promovam uma alimentação saudável vinculada a realização frequente de atividades físicas (Moura, et al., 2012; Souza \& Fernandes, 2014; Martins, et al., 2015; Nunes, et al., 2016).

Barbieri e colaboradores (2012), ressaltam que embora o pré-natal aconteça de maneira abrangente há uma desarticulação grave das consultas de enfermagem, bem como do contato com diferentes agentes de saúde, entre o período pré-natal e o puerpério. Salientando, 
portanto, a necessidade da perpetuação do vínculo pré-estabelecido com o enfermeiro ao longo do período puerperal, para que a saúde da mãe e do neonato possa ser monitorada também nesta etapa, afim de assegurar a qualidade de vida de ambos e mitigar a mortalidade (Rodrigues, 2015).

A assistência adequada do enfermeiro durante a consulta pré-natal tem sido satisfatória na detecção precoce de patologias que potencialmente poderiam se desenvolver no decorrer da gestação, como a Síndrome Hipertensiva da Gravidez, a qual aumenta consideravelmente a ocorrência de pré-eclâmpsia e em casos graves coloca em risco a saúde da mãe e do feto (Abrahão, et al., 2020).

Nesta perspectiva, Abrahão e colaboradores (2020), enfatizam a imprescindibilidade da consulta pré-natal da enfermagem, bem como da atuação educativa dos enfermeiros para a promoção da saúde durante a gestação, minimizando os riscos predisponentes à medida que qualifica a assistência prestada.

Desta forma, a consulta em enfermagem no pré-natal deve ser compreendida como um instrumento importante na educação e promoção da saúde gestacional, a qual vem sendo aprimorada nos últimos anos, mas que ainda carece de aprimoramento, via capacitação e formação permanente dos enfermeiros que a compõe (Santos, 2011).

\section{Conclusão}

O material bibliográfico analisado aponta que a atuação dos enfermeiros na educação e na promoção de saúde no pré-natal tem sido aprimorada de forma satisfatória nos últimos anos, com estabelecimento de vínculos consistentes entre enfermeiro e gestante, ao longo da assistência prestada.

Contudo, ainda existe um caminho a ser percorrido para que os enfermeiros atuem plenamente em prol da qualificação da assistência pré-natal. Tal jornada demanda de aprimoramento cientifico e formação continua por parte dos profissionais e de maior reconhecimento do papel do enfermeiro obstetra pela sociedade.

Desta forma, sugerimos que trabalhos futuros sejam desenvolvidos no intuito de orientar o enfermeiro obstetra, acerca do seu papel ao longo do acompanhamento pré-natal, afim de qualificar sua formação e consequentemente a assistência prestada. 


\section{Referencias}

Abrahão, Â. C. M., Santos, R. F., Viana, S. R. D. G., Viana, S. M., \& Costa, C. S. C. (2020). Atuação do enfermeiro a pacientes portadoras de síndrome hipertensiva específica da gestação. Rev. Cient. da Esc. Estadual de Saúde Pública de Goiás" Candido Santiago", 6(1):51-63.

Alves, C. N., Wilhelm, L. A., Barreto, C. N., Santos, C. C. D., Meincke, S. M. K., \& Ressel, L. B. (2015). Cuidado pré-natal e cultura: uma interface na atuação da enfermagem. Escola Anna Nery, 19(2), 265-271.

Araújo, S. M., Silva, M. E. D., Moraes, R. C., \& Alves, D. S. (2013). A importância do prénatal e a assistência de enfermagem. Veredas Favip-Revista Eletrônica de Ciências, 3(2), 1-7.

Barbieri, Â., Fonseca, L. M., Ceron, M. I., \& Fedosse, E. (2012). Análise da atenção pré-natal na percepção de puérperas. Distúrbios da Comunicação, 24(1), 29-39.

Brasil. Ministério da Saúde. Ministério da Saúde (2014). Caderneta da Gestante.

Brasil. Ministério da Saúde, \& Brasil. Ministério da Saúde. (2012). Caderno de atenção básica $\mathrm{n}^{\circ}$ 32: atenção ao pré-natal de baixo risco.

BRASIL. Ministério da Saúde. (2000). Portaria no 569/GM, 1 de junho de 2000: Institui o Programa de Humanização no Pré-natal e Nascimento e Implantação de Centrais de Regulação Obstétrica e Neonatal.

Brasil. (2006). Secretaria de Atenção à Saúde. Departamento de Ações Programáticas e Estratégicas. Área técnica de Saúde da Mulher. Pré-natal e puerpério: Atenção humanizada e qualificada. Manual Técnico. Brasília, DF: Ministério da Saúde.

Brazil. Ministério da Saúde. (2005). Assistência integral à saúde da mulher: bases de ação programática (Vol. 6). Centro de documentação do Ministério da Saúde. 
Brasil. Federação Brasileira das Associações de Ginecologia e Obstetrícia. (2011). Urgências e emergências maternas: guia para diagnóstico e conduta em situações de risco de morte materna. In Urgências e emergências maternas: guia para diagnóstico e conduta em situações de risco de morte materna (pp. 118-118).

Cabral, F. B., Hirt, L. M., der Sand, V., \& Pacheco, I. C. (2013). Atendimento pré-natal na ótica de puérperas: da medicalização à fragmentação do cuidado. Revista da Escola de Enfermagem da USP, 47(2), 281-287.

Castro, D. F. A. D. (2010). Qualidade de vida de gestantes assistidas pela estratégia saúde da família. Tese de Doutorado, Universidade de São Paulo, São Paulo, São Paulo, Brasil.

Chaves, M. M. N., Farias, F. C. D. S. Á., Apostólico, M. R., Cubas, M. R., \& Egry, E. Y. (2011). Amamentação: a prática do enfermeiro na perspectiva da Classificação Internacional de Práticas de Enfermagem em Saúde Coletiva. Revista da Escola de Enfermagem da USP, 45(1), 199-205.

Costa, C. S. C., Vila, V. D. S. C., Rodrigues, F. M., Martins, C. A., \& Pinho, L. M. O. (2013). Características do atendimento pré-natal na Rede Básica de Saúde. Revista eletrônica de enfermagem, 15 (2), 516-522.

Costa, M., Marinho, C. R. P., \& Moraes, A. P. P. (2015). Assistência pré-natal: competências desempenhadas por enfermeiros em ESFs do vale do São Patrício-GO. Revista Eletrônica da Faculdade de Ceres, 4(1), 1-17.

Coutinho, T., Teixeira, M. T. B., Dain, S., Sayd, J. D., \& Coutinho, L. M. (2003). Adequação do processo de assistência pré-natal entre as usuárias do Sistema Único de Saúde em Juiz de Fora-MG. Revista Brasileira de Ginecologia e Obstetrícia, 25(10), 717-724.

Cruz, R. D. S. B. L. C., Caminha, M. D. F. C., \& Batista Filho, M. (2014). Aspectos históricos, conceituais e organizativos do pré-natal. Rev. bras. ciênc. saúde, 18 (1), 87-94.

Dias, R. A. (2014). A importância do pré-natal na atenção básica. Trabalho de conclusão de curso, Universidade Federal de Minas Gerais, Teófilo Otoni, Minas Gerais, Brasil. 
Duarte, S. J. H., \& Mamede, M. V. (2013). Ações do pré-natal realizadas pela equipe de enfermagem na atenção primária à saúde, Cuiabá. Ciencia y Enfermeria, 19(1), 117-129.

Durand, M. K., \& Heidemann, I. T. (2013). The promotion of women's autonomy during family health nursing consultations. Revista da Escola de Enfermagem da USP, 47(2), 288295.

Ferreira, M. A., Carneiro, A. M. C. B., Valente, M. M. Q. P., Cestari, V. R. F., Diógenes, M. A. R., \& Diógenes, L. M. M. B. (2016). Prevalência do diagnóstico de enfermagem nutrição desequilibrada em hipertensos. Cogitare Enfermagem, 21(3), 1-9.

Fernandes, B. D. C. (2016). Cuidado pré-natal e puerperal na rede atenção básica à saúde do estado de Goiás. Dissertação de mestrado, Universidade Federal de Goiás, Goiânia, Goiás, Brasil.

Gomes, C., \& Sorj, B. (2014). Corpo, geração e identidade: a Marcha das vadias no Brasil. Sociedade e Estado, 29(2), 433-447.

Holanda, S. M., Castro, R. C. M. B., Aquin, P. D. S., Pinheiro, A. K. B., Lopes, L. G., \& Martins, E. S. (2018). Influência da participação do companheiro no pré-natal: satisfação de primíparas quanto ao apoio no parto. Texto \& Contexto-Enfermagem, 27(2), 1-10.

Kassada, D. S., Marcon, S. S., \& Waidman, M. A. P. (2014). Percepções e práticas de gestantes atendidas na atenção primária frente ao uso de drogas. Escola Anna Nery, 18(3), 428-434.

Lima, A. P., \& de Paula Corrêa, Á. C. (2013). A produção de dados para o Sistema de Informação do Pré-Natal em unidades básicas de saúde. Revista da Escola de Enfermagem da USP, 47(4), 876-883.

Lima, S. S. Enfermagem no pré-natal de baixo risco na estratégia Saúde da Família. (2013) Aquichan, 13 (2), 261-9. 
Martins, Q. P. M., Ferreira, G. S. M., de Araújo Aragão, A. E., Gomes, F. M. A., de Araújo, L. M., \& Ferreira, F. I. S. (2015). Conhecimentos de gestantes no pré-natal: evidências para o cuidado de enfermagem. SANARE-Revista de Políticas Públicas, 14(2), 65-71.

Matuda, C. G., Aguiar, D. M. D. L., \& Frazão, P. (2013). Cooperação interprofissional e a Reforma Sanitária no Brasil: implicações para o modelo de atenção à saúde. Saúde e Sociedade, 22, 173-186.

Neme, B., \& Zugaib, M. (2006). Assistência pré-natal. Obstetrícia básica. $3^{a}$ ed. São Paulo: Sarvier, 104-19.

Nunes, J. T., Oliveira Gomes, K. R., Pacheco Rodrigues, M. T., \& Medeiros Mascarenhas, M. D. (2016). Qualidade da assistência pré-natal no Brasil: revisão de artigos publicados de 2005 a 2015. Cadernos Saúde Coletiva, 24(2), 252-261.

ONU. Organização Das Nações Unidas. Declaração do novo milênio: Metas para o novo milênio. 2000.

Paraná, 2017. Linha guia rede mãe paranaense. Recuperado de https://www.saude.pr.gov.br/Pagina/Mae-Paranaense.

Pereira, A. S., Shitsuka, D. M., Parreira, F. J., \& Shitsuka, R. (2018). Metodologia da pesquisa científica.[e-book]. Santa Maria. Ed. UAB/NTE/UFSM. Recuperado de https://repositorio. ufsm. br/bitstream/handle/1/15824/Lic_Computacao_MetodologiaPesquisa-Cientifica. pdf.

Queiroz, D. J. M., Soares, D. B., \& de Oliveira, K. C. A. N. (2015). Avaliação da assistência pré-natal: relevância dos exames laboratoriais. Revista Brasileira em Promoção da Saúde, 28(4), 504-512.

Rios, C. T. F., \& Vieira, N. F. C. (2007). Ações educativas no pré-natal: reflexão sobre a consulta de enfermagem como um espaço para educação em saúde. Ciência \& Saúde Coletiva, 12, 477-486. 
Rocha, P. A., Soares, T. C., Farah, B. F., \& de Castro Friedrich, D. B. (2012). Promoção da saúde: a concepção do enfermeiro que atua no programa saúde da família. Revista Brasileira em Promoção da Saúde, 25(2), 215-220.

Rodrigues, I. R. (2015). Consulta de enfermagem no pré-natal: representações sociais de gestantes e enfermeiros. Dissertação de mestrado, Universidade Estadual Do Ceará, Fortaleza, Ceará.

Santos, P. D. (2011). Consulta de enfermagem no pré-natal: uma revisão de literatura.

Serruya, S. J., Lago, T. D. G., \& Cecatti, J. G. (2004). O panorama da atenção pré-natal no Brasil e o Programa de Humanização do Pré-natal e Nascimento. Revista Brasileira de Saúde Materno Infantil.

Silva, A. A. (2018). Pré-Natal da gestante de risco habitual: potencialidades e fragilidades na consulta. Trabalho de Conclusão do Curso, Universidade Federal de Minas Gerais, Araçuí, Minas Gerais, Brasil.

Silva, S. C., de Souza, K. V., Alves, V. H., \& Corrêa, B. A. (2016). Atuação do enfermeiro na consulta pré-natal: limites e potencialidades. Revista de Pesquisa Cuidado é Fundamental Online, 8(2), 4087-4098.

Souza, V. B., Roecker, S., \& Marcon, S. S. (2011). Ações educativas durante a assistência pré-natal: percepção de gestantes atendidas na rede básica de Maringá-PR. Revista Eletrônica de Enfermagem, 13(2), 199-210.

Souza, A. B. Q., \& Fernandes, B. M. (2014). Diretriz para assistência de enfermagem: ferramenta eficaz para a promoção da saúde no puerpério. Revista da Rede de Enfermagem do Nordeste, 15(4), 594-604.

Spindola, T., Progianti, J. M., \& Garcia Penna, L. H. (2012). Opinión de las gestantes sobre el acompañamiento de la enfermera obstetra en el prenatal de un hospital universitario. Ciencia y enfermería, $18(2), 65-73$. 
Vasconcelos - Moura, M. A., Fernandes, G., Santos, C., \& de Araújo-Silva, V. M. (2014). La calidad de asistencia de enfermería en el periodo prenatal desde la perspectiva de la mujer embarazada. Aquichan, 14(2), 196-206.

Viellas, E. F., Domingues, R. M. S. M., Dias, M. A. B., Gama, S. G. N. D., Theme Filha, M. M., Costa, J. V. D., ... \& Leal, M. D. C. (2014). Assistência pré-natal no Brasil. Cadernos de Saúde Pública, 30, S85-S100.

Viellas, E. F., Gama, S. G. N. D., Theme Filha, M. M., \& Leal, M. D. C. (2012). Gravidez recorrente na adolescência e os desfechos negativos no recém-nascido: um estudo no município do Rio de Janeiro. Revista Brasileira de Epidemiologia, 15, 443-454.

\section{Porcentagem de contribuição de cada autor no manuscrito}

Ana Alice Bueno Da Silva - 50\%

Claudiane Andrade - 50\% 